\title{
Temozolomide chemotherapy alone versus radiotherapy alone for malignant astrocytoma in the elderly: the NOA-08 randomised, phase 3 trial
}

Wick, W ; Platten, M ; Meisner, C ; Felsberg, J ; Tabatabai, G ; Simon, M ; Nikkhah, G ; Papsdorf, K ; Steinbach, J P ; Sabel, M ; Combs, S E ; Vesper, J ; Braun, C ; Meixensberger, J ; Ketter, R ; Mayer-Steinacker, R ; Reifenberger, G ; Weller, M

\begin{abstract}
BACKGROUND: Radiotherapy is the standard care in elderly patients with malignant astrocytoma and the role of primary chemotherapy is poorly defined. We did a randomised trial to compare the efficacy and safety of dose-dense temozolomide alone versus radiotherapy alone in elderly patients with anaplastic astrocytoma or glioblastoma. METHODS: Between May 15, 2005, and Nov 2, 2009, we enrolled patients with confirmed anaplastic astrocytoma or glioblastoma, age older than 65 years, and a Karnofsky performance score of 60 or higher. Patients were randomly assigned $100 \mathrm{mg} / \mathrm{m}(2)$ temozolomide, given on days 1-7 of 1 week on, 1 week off cycles, or radiotherapy of $60 \cdot 0$ Gy, administered over 6-7 weeks in 30 fractions of $1 \cdot 8-2 \cdot 0$ Gy. The primary endpoint was overall survival. We assessed non-inferiority with a $25 \%$ margin, analysed for all patients who received at least one dose of assigned treatment. This trial is registered with ClinicalTrials.gov, number NCT01502241. FINDINGS: Of 584 patients screened, we enrolled 412. 373 patients (195 randomly allocated to the temozolomide group and 178 to the radiotherapy group) received at least one dose of treatment and were included in efficacy analyses. Median overall survival was $8 \cdot 6$ months $(95 \%$ CI $7 \cdot 3-10 \cdot 2)$ in the temozolomide group versus $9 \cdot 6$ months $(8 \cdot 2-10 \cdot 8)$ in the radiotherapy group (hazard ratio [HR] $1 \cdot 09,95 \%$ CI $0 \cdot 84-1 \cdot 42, \mathrm{p}$ (non-inferiority) $=0 \cdot 033)$. Median event-free survival (EFS) did not differ significantly between the temozolomide and radiotherapy groups $(3 \cdot 3$ months [95\% CI $3 \cdot 2-4 \cdot 1]$ vs $4 \cdot 7[4 \cdot 2-5 \cdot 2]$; HR $1 \cdot 15,95 \%$ CI $0 \cdot 92-1 \cdot 43, \mathrm{p}$ (non-inferiority) $=0 \cdot 043)$. Tumour MGMT promoter methylation was seen in $73(35 \%)$ of 209 patients tested. MGMT promoter methylation was associated with longer overall survival than was unmethylated status $(11 \cdot 9$ months [95\% CI $9 \cdot 0$ to not reached] vs $8 \cdot 2$ months $[7 \cdot 0-$ $10 \cdot 0]$; HR $0 \cdot 62,95 \%$ CI $0 \cdot 42-0 \cdot 91, \mathrm{p}=0 \cdot 014)$. EFS was longer in patients with MGMT promoter methylation who received temozolomide than in those who underwent radiotherapy $(8 \cdot 4$ months $[95 \mathrm{e} \%$ CI $5 \cdot 5-11 \cdot 7]$ vs $4 \cdot 6[4 \cdot 2-5 \cdot 0])$, whereas the opposite was true for patients with no methylation of the MGMT promoter $(3 \cdot 3$ months $[3 \cdot 0-3 \cdot 5]$ vs $4 \cdot 6$ months $[3 \cdot 7-6 \cdot 3])$. The most frequent grade $3-4$ intervention-related adverse events were neutropenia (16 patients in the temozolomide group vs two in the radiotherapy group), lymphocytopenia (46 vs one), thrombocytopenia (14 vs four), raised liver-enzyme concentrations (30 vs 16), infections (35 vs 23 ), and thromboembolic events (24 vs eight). INTERPRETATION: Temozolomide alone is non-inferior to radiotherapy alone in the treatment of elderly patients with malignant astrocytoma. MGMT promoter methylation seems to be a useful biomarker for outcomes by treatment and could aid decision-making. FUNDING: Merck Sharp Dohme.
\end{abstract}

DOI: https://doi.org/10.1016/S1470-2045(12)70164-X 
Accepted Version

Originally published at:

Wick, W; Platten, M; Meisner, C; Felsberg, J; Tabatabai, G; Simon, M; Nikkhah, G; Papsdorf, K; Steinbach, J P; Sabel, M; Combs, S E; Vesper, J; Braun, C; Meixensberger, J; Ketter, R; Mayer-Steinacker, R; Reifenberger, G; Weller, M (2012). Temozolomide chemotherapy alone versus radiotherapy alone for malignant astrocytoma in the elderly: the NOA-08 randomised, phase 3 trial. Lancet Oncology, 13(7):707-715.

DOI: https://doi.org/10.1016/S1470-2045(12)70164-X 


\section{The NOA-08 randomized phase III trial of chemotherapy versus radiotherapy for malignant astrocytoma in the elderly}

Wolfgang Wick, MD, Prof. ${ }^{1,3,5}$, Michael Platten, MD, Prof. ${ }^{1,3,5}$, Christoph Meisner, $\mathrm{PhD}^{4}$, Jörg Felsberg, MD ${ }^{11}$, Ghazaleh Tabatabai, MD,PhD ${ }^{5,15}$, Matthias Simon, $\mathrm{MD}^{6}$, Guido Nikkhah, MD, Prof. ${ }^{7}$, Kirsten Papsdorf, MD ${ }^{8}$, Joachim P. Steinbach, MD, Prof. ${ }^{5,10}$, Michael Sabel; $M D^{11}$, Stephanie E. Combs, $M D^{2}$, Jan Vesper, $M D^{7,11}$, Christian Braun, $M D^{5}$, Jürgen Meixensberger, MD, Prof. ${ }^{9}$, Ralf Ketter, $\mathrm{MD}^{13}$, Regine Mayer-Steinacker, $\mathrm{MD}^{14}$, Guido Reifenberger, MD, Prof. ${ }^{12}$, Michael Weller, MD, Prof. ${ }^{5,15}$, for the NOA-08 Study Group* of the Neurooncology Working Group (NOA) of the German Cancer Society

${ }^{1}$ Departments of Neurooncology and ${ }^{2}$ Radiation Oncology, National Centre for Tumour Diseases, University of Heidelberg, Im Neuenheimer Feld 400, and ${ }^{3}$ Clinical Cooperation Unit Neurooncology, German Cancer Research Centre, D-69120 Heidelberg; ${ }^{4}$ Departments of Medical Biometry, Westbahnhofstraße 55, 72070 Tübingen and ${ }^{5}$ General Neurology and Hertie Institute for Clinical Brain Research, University of Tübingen, Hoppe-Seyler-Strasse 3, D-72076 Tübingen; ' Department of Neurosurgery, University of Bonn, Sigmund-FreudStrasse 25, D-53105 Bonn; ${ }^{7}$ Department of Stereotactic Neurosurgery, University Clinic Freiburg, Breisacher Strasse 64, D-79106 Freiburg; ${ }^{8}$ Departments of Radiation Oncology and ${ }^{9}$ Neurosurgery, University Hospital Leipzig, Stephanstrasse 9a, D-04103 Leipzig; ${ }^{10} \mathrm{Dr}$. Senckenbergisches Institute for Neurooncology, University of Frankfurt, Schleusenweg 2-16, D-60528 Frankfurt; ${ }^{11}$ Departments of Neurosurgery and ${ }^{12}$ Neuropathology, Heinrich Heine University, Moorenstrasse 5, D-40225 Düsseldorf; ${ }^{13}$ Department of Neurosurgery, University of Homburg, Kirrberger Strasse, D-66421 Homburg/Saar; ${ }^{14}$ Department of Internal Medicine III, University of Ulm, Albert-Einstein-Allee 23, D-89081 UIm, all Germany; ${ }^{15}$ Department of Neurology, University Hospital Zurich, Frauenklinikstrasse 26, CH-8091 Zurich, Switzerland. *Authors of the Study Group are mentioned separately.

\section{Address correspondence to}

Wolfgang Wick, MD

Department of Neurooncology, Neurology Clinic \& National Centre for Tumour Disease University of Heidelberg

Im Neuenheimer Feld 400, D-69120 Heidelberg, Germany

Tel.: $+49(0) 6221 / 56-7075$

Fax: $+49(0) 6221 / 56-7554$

E-mail: wolfgang.wick@med.uni-heidelberg.de 


\section{ABSTRACT}

\section{Background}

While radiotherapy (RT) is standard-of-care in elderly patients with malignant astrocytoma, the role of primary chemotherapy is poorly defined. The NOA-08 trial compared efficacy and safety of RT to temozolomide (TMZ) in patients with anaplastic astrocytoma (AA) or glioblastoma (GB).

\section{Methods}

Patients $(\mathrm{N}=412 ; 39 \mathrm{AA}, 373 \mathrm{~GB})>65$ years with a Karnofsky performance score $\geq 60$ were randomized in electronically generated blocks of variable length without stratification to receive standard RT to 60 Gy in $30 \times 2$ Gy fractions or TMZ in a one week on/one week off schedule. The primary endpoint was overall survival (OS). This trial (German Cancer Trials Registry ID 386 and NCT01502241) sought to demonstrate non-inferiority with a $25 \%$ margin of dose-dense TMZ compared with RT in the intention-to-treat population. The trial finished enrolment Nov 2 2009. Toxicity was graded according to the Common Terminology Criteria for Adverse Events (CTCAE), version 3.0.

\section{Findings}

Patient characteristics in the intention-to-treat population [N=373 (178 patients $R T, 195$ patients TMZ)] were balanced. Median OS [8.6 [7·3-10·2] months versus $9 \cdot 6[8 \cdot 2-10 \cdot 8]$ months; hazard ratio $(\mathrm{HR})=1.09(95 \% \mathrm{Cl}$ : 0.84-1.42)] of TMZ versus RT did not differ between both arms. Non-inferiority of TMZ compared with RT was significant $(p=0 \cdot 033)$. Also median event-free survival (EFS) [3.3 [3.2-4.1] months versus $4 \cdot 7[4 \cdot 2-5 \cdot 2]$ months did not differ with a HR=1.15 $(0.92-1 \cdot 43)]$ indicating non-inferiority $(p=0.043)$. DNA repair protein $O^{6}$ methylguanine DNA-methyltransferase (MGMT) promoter methylation in tumor tissue (73/209 patients, 34.9\%) tested was associated with prolonged OS [11.9 [9-not reached] versus $8 \cdot 2$ [7-10] months; $\mathrm{HR}=0 \cdot 67(0 \cdot 38-1 \cdot 29), \mathrm{p}=0 \cdot 0137]$. Patients with $M G M T$ promoter methylation had longer EFS when treated with TMZ (8.4 months [5.5-11.7] versus RT $(4 \cdot 6$ [4-2-5] months) whereas patients without MGMT promoter methylation had longer EFS when 
treated with RT (4.6 [3.7-6.3] versus $3 \cdot 3$ [3-3.5] months). The most common interventionrelated adverse events in each group were leukocytopenia, lymphocytopenia and thrombocytopenia (grade $3 / 4$ according to CTCAE: 12/4, 24/2, and 12/2 in the TMZ and 2, 1, and 4 in the RT arm), liver enzyme elevation (grade 3/4: 26/4 in the TMZ and 12/4 in the RT arm), infections (grade3/4: 26/9 in the TMZ and 15/8 in the RT arm), and thromboembolic events (grade $3 / 4: 18 / 6$ in the TMZ and $1 / 4$ in the RT arm).

Interpretation

NOA-08 demonstrates the non-inferiority of TMZ compared with RT in the treatment of elderly patients with malignant astrocytoma. To improve EFS, MGMT promoter methylation is a strong predictive biomarker for the choice between RT and TMZ.

\section{Funding}

Merck, Sharp \& Dohme

Keywords: glioma, radiotherapy, temozolomide, MGMT 


\section{INTRODUCTION}

Gliomas account for half of all intrinsic brain tumours and glioblastoma (GB) of World Health Organization (WHO) grade IV, the most malignant variant of glioma, accounts for half of all gliomas. On a population level, median survival with GB may still be below 6 months and age is the most important therapy-independent prognostic factor (www.cbtrus.org). ${ }^{1}$ In a few years more than half of the patients with GB will be older than 65 years of age and thus be classified as elderly. ${ }^{2}$ In the elderly, anaplastic astrocytoma (AA) (WHO grade III), a less common and malignant type of glioma with an overall better prognosis, shares molecular features and poor outcome with GB.,

The current standard of care in elderly patients with GB or AA is resection or biopsy followed by involved-field radiotherapy (RT). ${ }^{5}$ The classical treatment schedule of RT consists of 60 Gy in 30 fractions of 2 Gy although a hypofractionated schedule, e.g., $15 \times 2.66 \mathrm{~Gy}^{7}$ is used in some centres. Concomitant and adjuvant radiochemotherapy with the alkylating agent temozolomide (TMZ) has become the standard of care in the younger population of GB patients. ${ }^{7}$ However, the benefit from TMZ is largely restricted to patients with tumours exhibiting promoter methylation of the $O^{6}$-methylguanine-DNA methyltransferase (MGMT) gene, which encodes a DNA repair protein associated with alkylator resistance ${ }^{8,9}$ However, the benefit derived from the addition of chemotherapy decreases with age ${ }^{10}$ and age per se is considered a risk factor for cognitive side effects from cranial irradiation. ${ }^{2}$ Moreover, the tolerability of combined modality treatment of RT plus TMZ in the elderly appears to be reduced. ${ }^{11}$ Accordingly, TMZ chemotherapy alone has been explored and found to be feasible in GB of the elderly. ${ }^{12,13}$ Finally, we had reported encouraging progression-free survival rates at six months in patients with recurrent glioblastoma treated with a one week on one week off regimen. ${ }^{14}$ These promising studies encouraged the German Neurooncology Working Group (NOA) in 2005 to conduct a randomized phase III trial (NOA08) to demonstrate that dose-dense $T M Z$ alone was not inferior to RT alone in the 
management of newly diagnosed GB or AA in the elderly (> 65 years) and to evaluate the role of $M G M T$ promoter methylation in this patient group. 


\section{PATIENTS AND METHODS}

\section{Patients}

Patients with de novo histologically confirmed AA or GB and > 65 years of age, Karnofsky performance score $(\mathrm{KPS}) \geq 60$, no prior systemic chemotherapy or RT to the brain, and adequate bone marrow reserve, liver, and renal function were eligible. Inclusion into the trial was based on local diagnosis. Histologic diagnoses were confirmed centrally at the Brain Tumour Reference Centre, German Society for Neuropathology and Neuroanatomy, in Düsseldorf at study entry, according to the $\mathrm{WHO}$ classifications $2000^{15}$ and $2007^{16}$. Failure to confirm AA or GB would have resulted in exclusion from the intention-to-treat-population (ITT). Of note, there was no change in the diagnostic criteria for AA or GB between the two versions of the WHO classification.

\section{Trial Design and Treatment}

NOA-08 (German Cancer Trials Registry ID 386 and NCT01502241) was approved by the ethics committees (EC) of all 23 participating sites. The study was conducted from May 15 2005 through November 2 2010, with the last patient randomized on Nov 2 2009. The principal screening population consisted of patients who fulfilled the inclusion criteria histology, age and KPS. All study patients provided written informed consent. Patients were centrally randomized 1:1 to receive RT or TMZ in a one week on/one week off schedule (see Supplementary Information) (Fig. 1). At progression, treatment with TMZ in the RT arm and with $\mathrm{RT}$ in the TMZ arm was suggested in the protocol.

If toxicity in the TMZ arm resulted in delays longer than 4 weeks prior to six months of treatment, TMZ was stopped and RT was performed. Treatment was stopped at disease progression or for unacceptable toxicity according to the Common Terminology Criteria for Adverse Events (CTCAE), version 3.0.

\section{Randomization and Masking}


Sequence generation: Participant allocation was done according to an electronically generated randomization list in blocks of variable length without stratification. The sequence was generated prior to study start at the independent Contract Research Organization (CRO), alcedis (Gießen, Germany).

Allocation concealment. Enrolment was done at the study site by an investigator. Assignment was initiated by FAX transmission from the study site to the CRO for single patients fulfilling the eligibility criteria. A responsible project manager at the CRO performed the randomization process and reported the assignment to the trial group via FAX transmission to the study site.

Blinding and masking: Due to the procedures necessary for RT or TMZ treatment, blinding of investigators or participants was impossible. Similar the data had to be analysed with knowledge of the group assignment. Biases were prevented by strict adherence to an analysis plan that was written by the statistician (C.M) and approved by the lead investigators (W.W. and M.W.) prior to any analysis on the data.

\section{Evaluations}

Baseline examinations included physical examination, MRI, full blood cell counts, blood chemistry, Mini-Mental State Examination (MMSE), and quality-of-life questionnaire (QLQ) assessment with European Organisation for Research and Treatment of Cancer (EORTC) QLQ C-30 and BN-20. Patients received monthly clinical evaluation during therapies and a more comprehensive evaluation, which included MRI, MMSE, and QLQ 4 weeks after completing RT or 3 months after initiation of TMZ and every 3 months thereafter. In the RT arm full blood cell counts and blood chemistry were done at 4 weeks after starting RT. In the TMZ arm, full blood counts were done weekly and blood chemistry was done every 4 weeks during treatment. Toxicity was assessed biweekly. Tumour response or progression were defined according to Macdonald criteria $^{17}$ (see Supplementary Information). Importantly, an apparent increase in tumor volume or contrast-enhancement in the radiation field in the first scan post RT was not deemed a progression but resulted in re-MRI 4-6 weeks later. 


\section{Molecular Methods}

The MGMT promoter methylation status (see Supplementary Methods) was determined by two distinct methylation-specific PCR (MSP) assays. ${ }^{18,19}$ Real-time PCR-based quantitative testing on 182 samples revealing 152 conclusive results was done at MDX Health. ${ }^{19}$ These same samples plus 70 samples from stereotactic biopsies with another 57 conclusive results were evaluated by conventional MSP at the German Brain Tumor Reference Center. ${ }^{18}$ Where discrepancies were detected (in 4 samples; twice in each direction), the results from MDX Health were used.

\section{Statistical analysis}

The primary endpoint was overall survival (OS), measured in days from surgery to death. Secondary efficacy end points included EFS, best response, health-related quality of life (HRQOL) and safety. Event-free survival (EFS) was defined as time from surgery to first progression for patients with progression respectively to death for patients without progression. Patients without progression or death were censored at the day of the last contact. Univariate descriptive analysis of OS and EFS used Kaplan-Meier estimates ${ }^{20}$ and a Cox proportional hazard model for estimating Hazard Ratios (HR) with two-sided 95\%confidence intervals $(\mathrm{Cl})$ and median OS and EFS with two-sided 95\%-confidence intervals. The non-inferiority of TMZ compared to RT concerning OS and EFS was evaluated using a one-sided Log Rank test as described ${ }^{21,22}$ for a tolerance level of $-25 \%$ difference (radiotherapy group - TMZ group) in median OS respectively EFS. Only OS was analyzed for confirmatory analysis and - regarding the non-inferiority hypotheses based on Hazard ratio, decided for a one-sided significance level of 0.05.

The best responses ${ }^{17}$ in both arms were compared using Wilcoxon test. Multivariate analyses and HRQOL assessment ${ }^{23,24}$ is detailed in Supplementary Methods. All analyses of the primary and secondary efficacy endpoints were based on the intention-to-treat population, which included all randomized patients except patients who withdrew their 
consent for data analysis or patients who did not receive any dose of trial medication after randomization. The per-protocol analysis of the primary endpoint included only patients without major protocol violations; these were unconfirmed histological confirmation of diagnosis $(n=2)$ and age $\leq 65$ years $(n=9)$. The safety analyses concentrates on the documentation of adverse events (details in Supplementary Methods).

The sample size of the trial was based on the primary endpoint and the non-inferiority hypothesis with an equivalence/non-inferiority limit of - 53 days or $-25 \%$ of an assumed median OS of 7 months a one-sided significance level of $5 \%$, a recruitment duration of 48 months and a drop-out of $11 \%$.

Then, 412 subjects were found to be sufficient to achieve at least $80 \%$ power using the test procedure for testing the non-inferiority of $\mathrm{TMZ}$ for $\mathrm{OS} .^{21,22}$ Analyses were performed with Statistical Analysing Program SAS 9.1.3 (SAS Institute, Cary, NC). Alpha error was set to $5 \%$ for all tests in this study. The data were documented during the study into the study documentation RDE system of Alcedis (Gießen, Germany). Alcedis monitored the data quality.

This trial is registered as an International Standard Randomised Controlled Trial at the German Cancer Trials Registry (ID 386, quality level A) and with ClinicalTrials.gov (NCT01502241).

\section{Role of the funding sources}

The funding source, former Schering Plough, now Merck, Sharp \& Dohme, had no role in the study design, collection, analysis, or interpretation of the data or in writing the study report or manuscript. Access to the raw data was limited to W.W., C.M., J.F., G.R. and M.W. The corresponding author had full access to all of the data and the final responsibility to submit the publication. 


\section{RESULTS}

\section{Patient characteristics}

The ITT population consisted of 373 elderly patients with centrally reviewed AA (11\%) or GB (89\%) who were randomized between May 152005 and November 22009 and received at least one dose of dose-dense TMZ or one fraction of RT. The per-protocol population consisted of 362 patients. The mean age of the ITT patients was 71 (RT arm) and 72 (TMZ arm) years, and risk factors were well balanced. Of note, there was a tendency to use steroids when RT was administered (Table 1). A total of 149 patients completed RT, and 126 patients in the TMZ group completed at least 4 cycles (8 weeks) of chemotherapy. Patients in both arms had similar frequencies of salvage therapies (74 patients $(70 \%)$ in the RT arm and 88 patients $(62 \%)$ in the TMZ arm), which mainly consisted of TMZ in the RT arm and vice versa (Supplementary Table 1). The likelihood of receiving salvage therapy did not differ between patients with MGMT promoter methylated or unmethylated tumors (data not shown).

\section{Tolerability and toxicity}

In general, most patients tolerated both treatments well. There were no CTCAE grade 5 toxicities in this study. CTCAE grade 2 - 4 toxicities were more frequent in the TMZ arm in all categories except for cutaneous AE (Table 2). The main reasons for discontinuation of RT were disease progression $(n=10)$ and prolonged infection $(n=8)$. The median number of TMZ cycles in arm B was 5 (range: $0-20)$. Discontinuations occurred due to progression $(n=141)$ or toxicity $(\mathrm{n}=28)$.

\section{Clinical Efficacy}

At a minimal follow-up of 12 months (median $25 \cdot 2$ months [20.0-not reached (NR)]) after the last patients had been randomized, 228 deaths have been observed in the first year (RT: 107, TMZ: 121). The estimation for the 1-year-OS rate was $37 \cdot 4 \%(95 \%-\mathrm{Cl}: 30 \cdot 2 \%-44 \cdot 7 \%)$ 
in the RT arm with a median OS of $9 \cdot 6$ months [95\%-CI: $8 \cdot 2-10 \cdot 8]$. The TMZ group had an estimated 1-year OS of $34.4 \%(95 \%-\mathrm{Cl}: 27 \cdot 6 \%-41 \cdot 4 \%)$ and a median survival of $8 \cdot 6$ months [95\%-Cl: $7 \cdot 3-10 \cdot 2]$. The non-inferiority of $\mathrm{TMZ}$ in comparison to RT for a tolerance level of $25 \%$ was statistically confirmed (Wellek test procedure, tolerance-level 25\%: $\mathrm{p}=0.033)^{21,22}$ with a HR of $1.1(95 \%-\mathrm{Cl}: 0.84-1 \cdot 42)($ Table 3 and Fig. $2 a)$. The non-inferiority of TMZ was also confirmed in the analysis of the per-protocol population (Wellek test procedure, tolerance level $25 \%: p=0 \cdot 028$ ).

Three-hundred-twenty-five patients experienced an event (progression or death) within in the first 12 months after surgery. There was no evidence for pseudoprogressions. The estimate for the 1-year-EFS rate was $9.3 \%(95 \%-\mathrm{Cl}: 5 \cdot 5 \%-14 \cdot 3 \%)$ in the RT arm with a median EFS of $4 \cdot 7$ months [95\%-Cl: $4 \cdot 2-5 \cdot 2]$ and 156 events. The TMZ group had an estimated 1-year EFS of $12.0 \%(95 \%-\mathrm{Cl}: 7 \cdot 8 \%-17 \cdot 1 \%)$ and a median EFS of $3 \cdot 3$ months [95\%-Cl: $3 \cdot 2-4 \cdot 1$ ] and 169 events, which comprised toxicities and deaths without documented progression (Wellek test procedure, tolerance-level 25\%: $p=0.043)^{20,21}$ with a HR of $1.15(95 \%-\mathrm{Cl}$ : $0.92-$ 1.43) (Table 3 and Fig. 2b). The non-inferiority of TMZ for EFS was also confirmed in the analysis of the per-protocol population (Wellek test procedure, tolerance level 25\%: $p=0 \cdot 041)^{21,22}$.

In the RT arm 106 patients experienced a progression. Seventy-four patients (69.8\%) received salvage therapy, whereas 88 of 141 patients $(62 \cdot 4 \%)$ with progression received salvage therapy in the TMZ arm $(p=0 \cdot 227)$. There was a higher likelihood of second surgery in the TMZ arm $[p=0.102$; relative risk $1.6(95 \% \mathrm{Cl}: 0.9-2 \cdot 9)]$. Otherwise, the patients in both arms had a similar frequency of salvage therapy, mainly TMZ in the RT arm, and vice versa (Supplementary Table 1).

\section{Prognostic and Predictive Factors}

MGMT promoter methylation was analysed for a subgroup of 209 patients (Table 1). This subgroup was comparable to the group of 165 patients without MGMT promoter methylation status concerning the distribution of type of primary surgery, histology, age and therapy 
group. MGMT promoter methylation was detected in 73 of 209 evaluable patients $(35 \%)$ (Table 1). This value was also detected in the subgroup of stereotactic biopsies with 20 of 59 tested samples showing a MGMT promoter methylation (33.8\%). The patients tested are representative for the ITT population in all relevant aspects and stereotactic biopsies resembled overall testing results (data not shown). Extent of resection (complete versus incomplete or biopsy; incomplete versus biopsy), and MGMT promoter methylation in tumor tissue, but not age in years neither as a continuous variable or dichotomized at age 70 , or histology (AA versus $\mathrm{GB}$ ) was found to be an independent prognostic factor for OS in the multivariate Cox analysis. An interaction was found between MGMT promoter methylation (methylated versus unmethylated) and therapy (Table 4a, Fig. 2c). These results were found also for EFS (Table 4b, Fig. 2d).

Importantly, MGMT promoter methylation was associated with improved EFS only in the TMZ arm, but not in the RT arm (Fig. 2e,f, Table 4, Supplementary Table 2). In the TMZ arm, median EFS for patients with a methylated MGMT promoter was 8.5 months $(95 \%-\mathrm{Cl} 5 \cdot 6$ $11 \cdot 9$ days) compared with 3 months (95\%-Cl 2.6-3.3 days) for patients with an unmethylated MGMT promoter (Fig. $2 f$ and Supplementary Table 2b). In contrast, MGMT promoter methylation did not influence EFS in the RT arm. However, in patients with a methylated MGMT promoter EFS (and with a trend for OS) was worse with RT than with TMZ while in patients with an unmethylated MGMT promoter EFS (and with a trend for OS) was superior with RT than with TMZ (Fig. 2e,f and Supplementary Table 2b).

\section{Quality-of-Life Assessments}

HRQOL was comparable in both groups and available from $82 \%$ of all patients. No clinically meaningful or statistically remarkable difference between the two groups over time in any of the scales or cohorts were observed in any of the three cohorts, except more discomfort from communication deficits in the RT arm for patients who died between 6 and 12 months $(p=0 \cdot 002)$. Supplementary Figure 1 shows the trajectories of the HRQOL mean scores. 


\section{DISCUSSION}

NOA-08 broadens the spectrum of primary treatment of elderly patients with malignant gliomas by demonstrating the non-inferiority of primary treatment of elderly patients with malignant gliomas with TMZ alone. It implements MGMT promoter methylation as a relevant biomarker to decide, when patients may be undertreated with primary RT alone.

The current standard of care for the increasing population of elderly patients with GB or AA is surgery or biopsy followed by RT. ${ }^{5}$ While RT alone is superior to best supportive care in elderly patients with GB and does not reduce $\mathrm{QOL}^{5}$, age per se may be a risk factor for cognitive side effects of cranial irradiation ${ }^{2}$, yet solid evidence for such cognitive side effects only exists for younger patients with irradiated low-grade tumors. ${ }^{25}$ Whether the addition of chemotherapy to $\mathrm{RT}^{7}$ improves outcome in the elderly, too, is currently explored. Many elderly patients do not even receive chemotherapy at recurrence. ${ }^{26}$ To challenge this current practice, new trial data are needed. In a recent ANOCEF trial, elderly patients $>70$ years even with a low KPS $<70$ seemed to benefit from TMZ alone when compared to historical controls. ${ }^{27}$ The NOA-08 trial for the first time demonstrates that dose-dense TMZ followed by RT as salvage is an alternative option in this patient population that is not inferior to RT alone followed by TMZ as salvage therapy. Given the limited life expectancy, dose-dense TMZ may be particularly suited for patients who may not have easy access to a radiation oncology facility and who prefer an oral medication administered and monitored close to home. However, a decline in cognitive functioning by RT should not be used for decisionmaking since neither the NOA-08 data nor a previously published trial ${ }^{5}$ support a relevance of this presumed unwanted effect (Table 1 and Supplementary Fig. 1), although formal neurocognitive testing was not performed as a meaningful differentiation between the in part rapid disease progression (Table 3 , supplementary Table $2 b$ ) and an evolving neurocognitive deficit was regarded impossible.

More importantly, the major novelty reported here is the strong predictive power of the MGMT status for the benefit to be expected from either treatment modality for EFS and as a yet non-significant trend for OS: MGMT promoter methylated tumours respond better to TMZ 
whereas unmethylated tumours respond better to RT (Fig. 2e,f, Table 4a,b). The data do not indicate that the non-significant effect for OS is due to resolving pseudoprogressions in the RT arm for methylated patients, but that these patients do better respond to the salvage TMZ treatment (Fig. 2e). The concept of pseudoprogression, although not the explicit terminology was well known at all study sites and regularly ruled out by short interval rescanning with MRI. Such stratification by a single biomarker has not been established in neurooncology despite supportive landmark data ${ }^{7,8}$ and is rare in general oncology, too. Accordingly, despite the acknowledged challenges associated with MGMT promoter methylation testing ${ }^{9}$, our data confirm the hypothesis generated by the recent cohort analysis of the German Glioma Network ${ }^{28}$, and in conjunction justify or even call for the routine testing of the MGMT promoter status in elderly patients with GB or AA, (i) to improve outcome, (ii) to prevent unnecessary toxicity and (iii) to save cost.

NOA-08 has its limitations, not only because of the inherent weaknesses of a non-inferiority design, the selection of a generous tolerance level, a one-sided test procedure and possible non-proportional hazards for EFS. Only $56 \%$ of the tissues were available and informative for MGMT testing. The limited number of informative results is mainly due to the high percentage of stereotactic biopsies (Table 1), which may not qualify for MGMT testing due to the limited amount of tumour DNA that can be extracted from these small tissue specimens. Still, both the results from the testing of stereotactic biopsies resembled overall testing results and the patients tested are representative for the ITT population in all relevant aspects. While TMZ exhibited haematological toxicity, liver enzyme elevations, asthenia/fatigue and gastrointestinal side effects in a relevant number of patients, more severe grade 4 toxicity was rare (Table 2). This toxicity may well be due to the intensified dosing schedule used in this trial and particularly careful to evaluate in this elderly patient population, where also grade 2 toxicities may impact quality of life. In a recent trial in younger patients with $\mathrm{GB}$, dose intensification in the adjuvant setting of primary combined modality treatment was not associated with increased OS, neither in patients with 
methylated nor with unmethylated MGMT promoter status. ${ }^{29}$ Thus, it may well be that TMZ alone can be given in a conventional schedule in elderly patients with MGMT promoter methylated malignant glioma with comparable efficacy as demonstrated in this trial but reduced toxicity. However, at the time this trial was designed and conducted, there were no data that compared different TMZ schedules, but only data indicative of a superior efficacy of the dose-dense TMZ schedule ${ }^{14}$, which also led to the design of the glioblastoma trial 0525 by the Radiation Therapy Oncology Group, which is reported to be negative. ${ }^{29}$ Similar, information on the potential non-inferiority of shorter courses of RT were only evolving, when NOA-08 already had been designed. ${ }^{6,30}$

The age cut-off of 65 years to define elderly patients remains a controversial issue in neurooncology and even arbitrary in some patients. ${ }^{2}$ While it may shift towards 70 in the field of $\mathrm{GB}$, it may shift to 60 in primary brain lymphoma where more aggressive treatments are explored. Other factors, such as neurological function or comorbidities are relevant, while in a generally well trial cohort such as the NOA-08 trial population, age alone is not prognostic any more (Table 4).

We propose that future research efforts should explore the biological basis underlying the poorer outcome associated with gliomas in the elderly and should step-wise replace age per se as a basis for clinical decision making. In this regard, the identification of IDH1 mutations as a positive prognostic marker restricted to gliomas of younger patients was a first important step. ${ }^{4} \mathrm{AA}$ in the elderly and primary GB both typically lack of $I D H 1$ mutations and show a similar, unfavourable outcome $e^{3,4}$, justifying the inclusion of AA patients in this trial and the focus on MGMT but not IDH testing. In the limited number of non-GB patients included (Table 1), however, we find that AA patients do better with both treatments (HR $0 \cdot 7$, Table 4), albeit worse than younger patients with AA in previous trials. ${ }^{31,32}$ In that respect, the IDH-associated glioma-CpG island methylator phenotype-related profiles ${ }^{32}$ may dilute the predictive properties of MGMT promoter methylation demonstrated in the NOA-08 trial, thus providing a possible explanation why MGMT lacks predictive properties in anaplastic glioma in younger patients. ${ }^{31}$ 
In summary, NOA-08 demonstrated that TMZ chemotherapy alone is not inferior to RT alone in elderly patients with newly diagnosed GB or AA. This practice-changing observation will be confirmed in the independent Nordic trial that also reported a similar efficacy of RT alone and TMZ, albeit in a different regimen of 5 days TMZ out of 28 days, and in patients with GB aged 61 or more..$^{30}$ More importantly, NOA-08 defines MGMT promoter methylation as a strong predictive biomarker that should help to guide clinicians to select among these two therapeutic options (Fig. 2 e,f and Supplementary Table 2). As a complementary approach and a next step of standardizing treatment of elderly patients with gliomas, the joint study of NCIC (CE.6) and EORTC (26062/22061) explores the efficacy of RT versus RT plus TMZ in elderly patients with GB. NOA-08 will certainly provoke a discussion on the standard-of-care arm, RT, in the methylated patients, especially since these patients do not shown a superior benefit from salvage TMZ compared to the unmethylated patients (Fig. 2e). Data from the $\mathrm{NCIC/EORTC}$ study may further validate the role of MGMT as a predictive biomarker in this patient population. It may confirm that TMZ produces no benefit in patients with unmethylated tumours, but, in case of a positive outcome, will provoke the question whether TMZ alone with deferred RT may be a sufficient treatment in patients with MGMT promoter methylated tumours. 


\section{REFERENCES}

1 Central Brain Tumor Registry of the United States: 2009-2010 CBTRUS Statistical Report: Primary Brain and Central Nervous System Tumors Diagnosed in Eighteen States in 2002-2006, 2009

2 Weller M, Platten M, Roth P, Wick W. Geriatric neuro-oncology: from mythology to biology. Curr Opin Neurol 2011; 24: 599-604.

3 Barnholtz-Sloan JS, Williams VL, Maldonado JL, et al. Patterns of care and outcomes among elderly individuals with primary malignant astrocytoma. J Neurosurg 2008; 108: 6428.

4 Hartmann C, Hentschel B, Wick W, et al. Patients with IDH1 wild type anaplastic astrocytomas exhibit worse prognosis than IDH1-mutated glioblastomas, and IDH1 mutation status accounts for the unfavorable prognostic effect of higher age: implications for classification of gliomas. Acta Neuropathol 2010; 120: 707-18.

5 Keime-Guibert F, Chinot O, Taillandier L, et al. Radiotherapy for glioblastoma in the elderly. N Engl J Med 2007; 356: 1527-35.

6 Roa W, Brasher PM, Bauman G, et al. Abbreviated course of radiation therapy in older patients with glioblastoma multiforme: a prospective randomized clinical trial. $J$ Clin Oncol 2004; 22: 1583-8.

7 Stupp R, Mason WP, van den Bent MJ, et al. Radiotherapy plus concomitant and adjuvant temozolomide for glioblastoma. N Engl J Med 2005; 352: 987-96.

8 Hegi ME, Diserens AC, Gorlia T, et al. MGMT gene silencing and benefit from temozolomide in glioblastoma. N Engl J Med 2005; 352: 997-1003.

9 Weller M, Stupp R, Reifenberger G, et al. MGMT promoter methylation in malignant gliomas: ready for personalized medicine? Nat Rev Neurol 2010; 6: 39-51.

10 Stupp R, Hegi ME, Mason WP, et al. Effects of radiotherapy with concomitant and adjuvant temozolomide versus radiotherapy alone on survival in glioblastoma in a randomised phase III study: 5-year analysis of the EORTC-NCIC trial. Lancet Oncol 2009; 10: 459-66.

11 Sijben AE, Mclntyre JB, Roldan GB, et al. Toxicity from chemoradiotherapy in older patients with glioblastoma multiforme. J Neurooncol 2008; 89: 97-103.

12 Glantz M, Chamberlain M, Liu Q, et al. Temozolomide as an alternative to irradiation for elderly patients with newly diagnosed malignant gliomas. Cancer 2003; 97: 2262-6.

13 Chinot OL, Barrie M, Frauger E, et al. Phase II study of temozolomide without radiotherapy in newly diagnosed glioblastoma multiforme in an elderly population. Cancer 2004; 100: 2208-14.

14 Wick W, Steinbach JP, Küker WM, Dichgans J, Bamberg M, Weller M. One week on/one week off: a novel active regimen of temozolomide for recurrent glioblastoma. Neurology 2004; 62:2113-5. 
15 Kleihues P, Cavenee WK. Pathology and genetics of tumours of the nervous system. Vol. 1 of World Health Organization classification of tumours. Lyon, France: IARC Press; 2000.

16 Louis DN, Ohgaki H, Wiestler OD et al. The 2007 WHO classification of tumours of the central nervous system. Acta Neuropathol 2007; 114: 97-109.

17 Macdonald DR, Cascino TL, Schold SC, Jr., Cairncross JG. Response criteria for phase II studies of supratentorial malignant glioma. J Clin Oncol 1990; 8: 1277-80.

18 Felsberg J, Rapp M, Loeser S, et al. Prognostic significance of molecular markers and extent of resection in primary glioblastoma patients. Clin Cancer Res 2009; 15: 6683-93.

19 Vlassenbroeck I, Califice S, Diserens AC, Migliavacca E, Straub J, Di Stefano I, Moreau F, Hamou MF, Renard I, Delorenzi M, Flamion B, DiGuiseppi J, Bierau K, Hegi ME. Validation of Real-Time MSP to Determine MGMT Promoter Methylation in Glioma. J Mol Diagn 2008; 10: 332-7.

20 Kaplan E, Meier P. Non-parametric estimation from incomplete observations. J Am Stat Assoc 1958; 53: 457-81.

21 Wellek S: Testing statistical hypotheses of equivalence. Chapman\&Hall 2003.

22 Wellek S: A Log-Rank Test for Equivalence of two Survivor Functions. Biometrics 49, $1993,877-881$

23 Taphoorn MJB, Stupp R, Coens C, et al. Health-related quality of life in patients with glioblastoma: a randomised controlled trial. Lancet Oncol 2005; 6: 937-44.

24 Kurland BF, Johnson LL, Egleston BL, Diehr PH. Longitudinal data with follow-up truncated by death: match the analysis method to research aims. Stat Sci 2009; 24: 211.

25 Douw L, Klein M, Fagel SS, et al. Cognitive and radiological effects of radiotherapy in patients with low-grade glioma: long-term follow-up. Lancet Neurol 2009; 8: 810-8.

26 Iwamoto FM, Reiner AS, Panageas KS, et al. Patterns of care in elderly glioblastoma patients. Ann Neurol 2008; 64: 628-34

27 Gállego Pérez-Larraya J, Ducray F, et al. Temozolomide in elderly patients with newly diagnosed glioblastoma and poor performance status: an ANOCEF phase II trial. J Clin Oncol 2011; 29: 3050-5.

28 Reifenberger G, Hentschel B, Felsberg J, et al. for the German Glioma Network. Predictive impact of MGMT promoter methylation in glioblastoma of the elderly. Int $J$ Cancer 2011 Dec 3.

29 Gilbert M, Mehta M, Aldape, K, Stupp R, Hegi M, Jaeckle K, Armstrong T, et al. RTOG 0525: A randomized phase III trial comparing standard adjuvant temozolomide with a dosedense schedule in newly diagnosed glioblastoma. J Clin Oncol 2011; 29: 2006.

30 Malmstrom A, Gronberg BH, Stupp R, et al. Glioblastoma (GBM) in elderly patients: A randomized phase III trial comparing survival in patients treated with 6-week radiotherapy (RT) versus hypofractionated RT over 2 weeks versus temozolomide single-agent chemotherapy (TMZ). J Clin Oncol 2010; 28:LBA 2002 (No 18 suppl (June 20 Supplement)). 
31 Wick W, Hartmann C, Engel C, et al. for the Neurooncology Working Group (NOA) of the German Cancer Society. NOA-04 randomized phase III trial of sequential radiochemotherapy of anaplastic glioma with PCV or temozolomide. J Clin Oncol 2009; 27: 5874-80.

32 van den Bent MJ, Gravendeel LA, Gorlia T et al. A hypermethylated phenotype in anaplastic oligodendroglial brain tumors is a better predictor of survival than MGMT methylation in anaplastic oligodendroglioma: a report from EORTC study 26951. Clin Cancer Res 2011 Sep 13 


\section{ACKNOWLEDGMENTS}

We are indebted to the patients and their families for agreeing to participate in this trial, as well as to the nurses and data managers for their collaboration. We would like to thank Julius Schuth, PharmD and Detlef Hecker, MD for their valuable scientific advice during conduct of the study.

The study has been awarded the quality label A by the German Cancer Society.

Alcedis (Giessen, Germany) served as CRO for monitoring and data collection.

The first round of MGMT testings reported in this study was done as a free scientific service by MDx Health (Liège, Belgium).

A preplanned event-driven analysis of the primary endpoint was presented at the ASCO Meeting 2010 (Wick W, Engel C, Combs SE et al. J Clin Oncol 2010 ASCO Annual Meeting Proceedings (Post-Meeting Edition) 2010;Vol 28:LBA 2001 (No 18 suppl (June 20 Supplement)) (for details see Supplementary Note).

CONFLICT OF INTERESTW.W., J.P.S, G.R. AND M.W. report on having received consulting and lecture fees from MSD. W.W. and M.W. have received research support from MSD.

M.P., C.M., J.F., G.T., M.S., G.N., K.P., M.S., S.E.C., J.V., C.B., J.M., R.K., and R.M.-S. declare no conflicts of interest.

\section{CONTRIBUTORS}

The concept of the trial was developed by M.W. and W.W. in collaboration with the German Neurooncology Working Group (NOA) in the German Cancer Society.

All data were collected by Alcedis and reviewed by W.W.

The statistical analyses were performed by C.M., a statistician at the Institute of Medical Biometry in Tübingen assisted by Rainer Stolper.

Histological specimens were reviewed centrally at the Brain Tumor Reference Centre of the German Society for Neuropathology and Neuroanatomy at the University of Düsseldorf Medical Centre by G.R. and J.F.

Analysis of the MGMT promoter methylation status was performed by MDx Health Care and by J.F. and G.R.

The article was written by W.W and M.W. with support from M.P., C.M., J.F., G.T., M.S., G.N., K.P., J.P.S., M.S., S.E.C., J.V., C.B., J.M., R.K., R.M.-S., G.R..

W.W., M.P., C.M., J.F., G.T., M.S., G.N., K.P., J.P.S., M.S., S.E.C., J.V., C.B., J.M., R.K., R.M.-S., G.R. and M.W. reviewed and approved the manuscript. 


\section{FIGURE LEGENDS}

Figure 1. Trial design and CONSORT flow chart. Patients were randomized $1: 1$ to receive RT or TMZ chemotherapy in an one week on/ one week off schedule. Patient numbers represent the intention-to-treat population. At progression, patients treated initially with RT were commonly treated with TMZ. Patients who progressed on or after TMZ were often treated with RT (Abbreviations: intention-to-treat population, ITT; radiotherapy, RT; temozolomide, TMZ).

Figure 2. Kaplan-Meier survival estimates. Data of OS (panel a) or EFS (panel b) were analyzed by treatment arm and tested for non-inferiority or by MGMT promoter methylation status (MGMT promoter methylated (MGMT+) or unmethylated (MGMT-), panels $\mathrm{c}$ and d) and tested for difference. Of note, Fig. $2 b$ is presenting non-proportional curves, which are deemed unproblematic in the context of non-inferiority. Data of OS (panel e) or EFS (panel f) of the TMZ- or RT-treated patients were also analyzed by MGMT promoter methylation status (MGMT promoter methylated (MGMT+) or unmethylated (MGMT-) and tested for difference. 
Figure 1

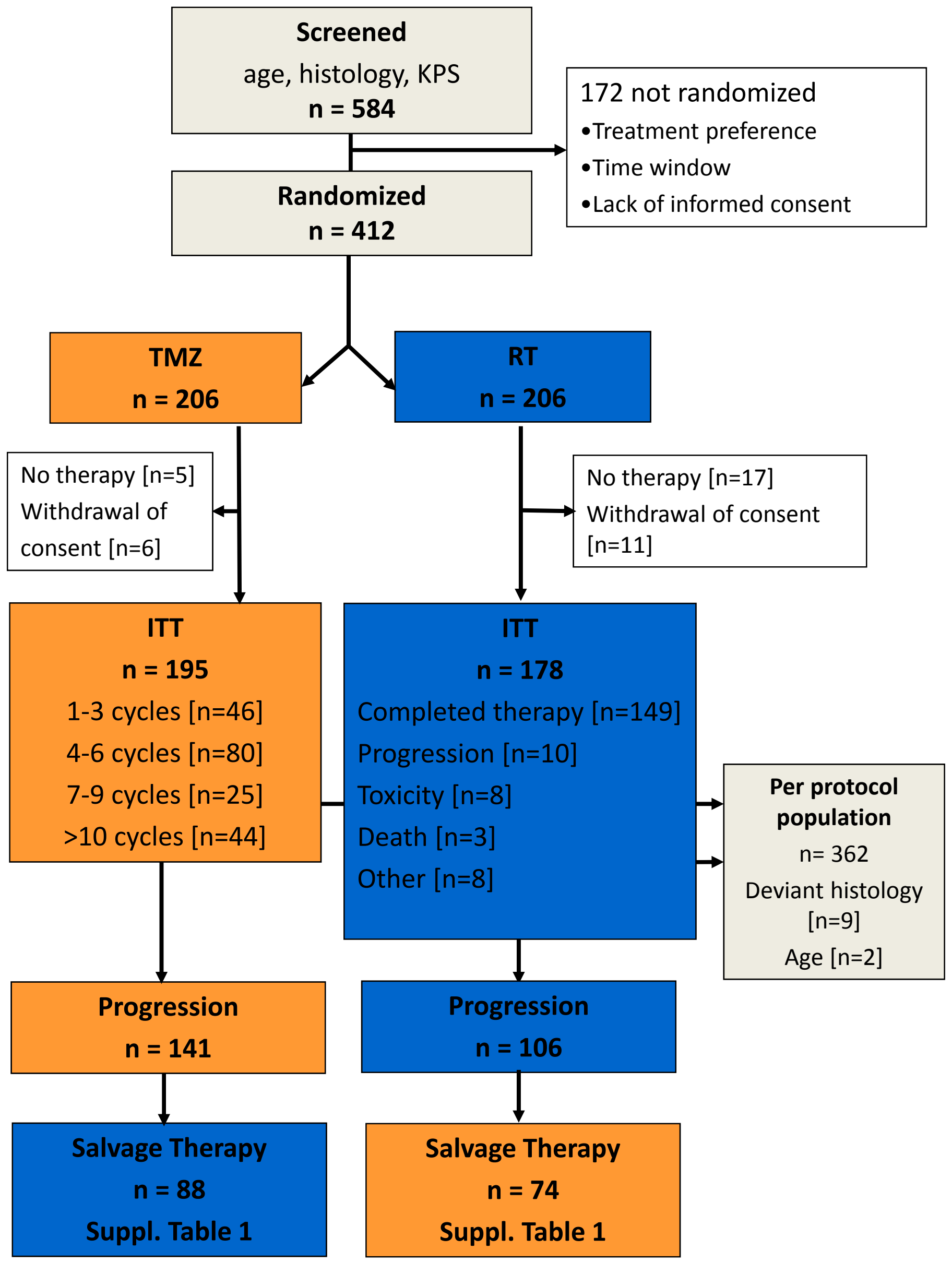




\section{Figure 2}

a

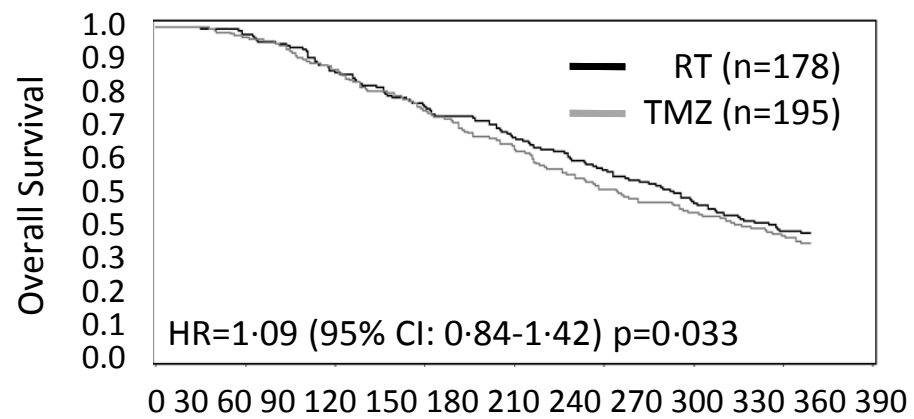

Time in Days

\begin{tabular}{|c|c|c|c|c|c|c|c|c|c|c|c|c|c|}
\hline $\begin{array}{l}\text { Time/ } \\
\text { Pts at } \\
\text { risk }\end{array}$ & 30 & 60 & 90 & 120 & 150 & 180 & 210 & 240 & 270 & 300 & 330 & 360 & 390 \\
\hline $\begin{array}{l}\text { RT } \\
178\end{array}$ & 176 & $\begin{array}{l}16 \\
8\end{array}$ & 154 & 142 & 130 & 123 & 110 & 100 & 90 & 79 & 67 & 59 & 53 \\
\hline $\begin{array}{l}\text { TMZ } \\
195\end{array}$ & 194 & $\frac{18}{7}$ & 170 & 152 & 140 & 122 & 111 & 96 & 85 & 78 & 69 & 60 & 52 \\
\hline
\end{tabular}

\section{b}

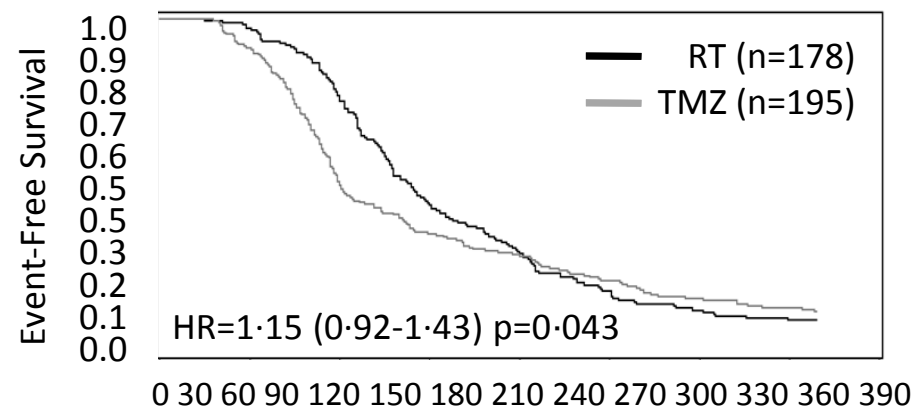

Time in Days

\begin{tabular}{|c|c|c|c|c|c|c|c|c|c|c|c|c|c|}
\hline $\begin{array}{l}\text { Time/ } \\
\text { Pts at } \\
\text { risk }\end{array}$ & 30 & 60 & 90 & 120 & 150 & 180 & 210 & 240 & 270 & 300 & 330 & 360 & 390 \\
\hline $\begin{array}{l}\text { RT } \\
178\end{array}$ & 176 & $\begin{array}{l}16 \\
4\end{array}$ & 145 & 108 & 75 & 62 & 41 & 34 & 24 & 21 & 16 & 15 & 10 \\
\hline $\begin{array}{l}\text { TMZ } \\
195\end{array}$ & 194 & $\begin{array}{l}16 \\
8\end{array}$ & 119 & 83 & 68 & 58 & 50 & 42 & 33 & 29 & 25 & 23 & 22 \\
\hline
\end{tabular}


Figure 2
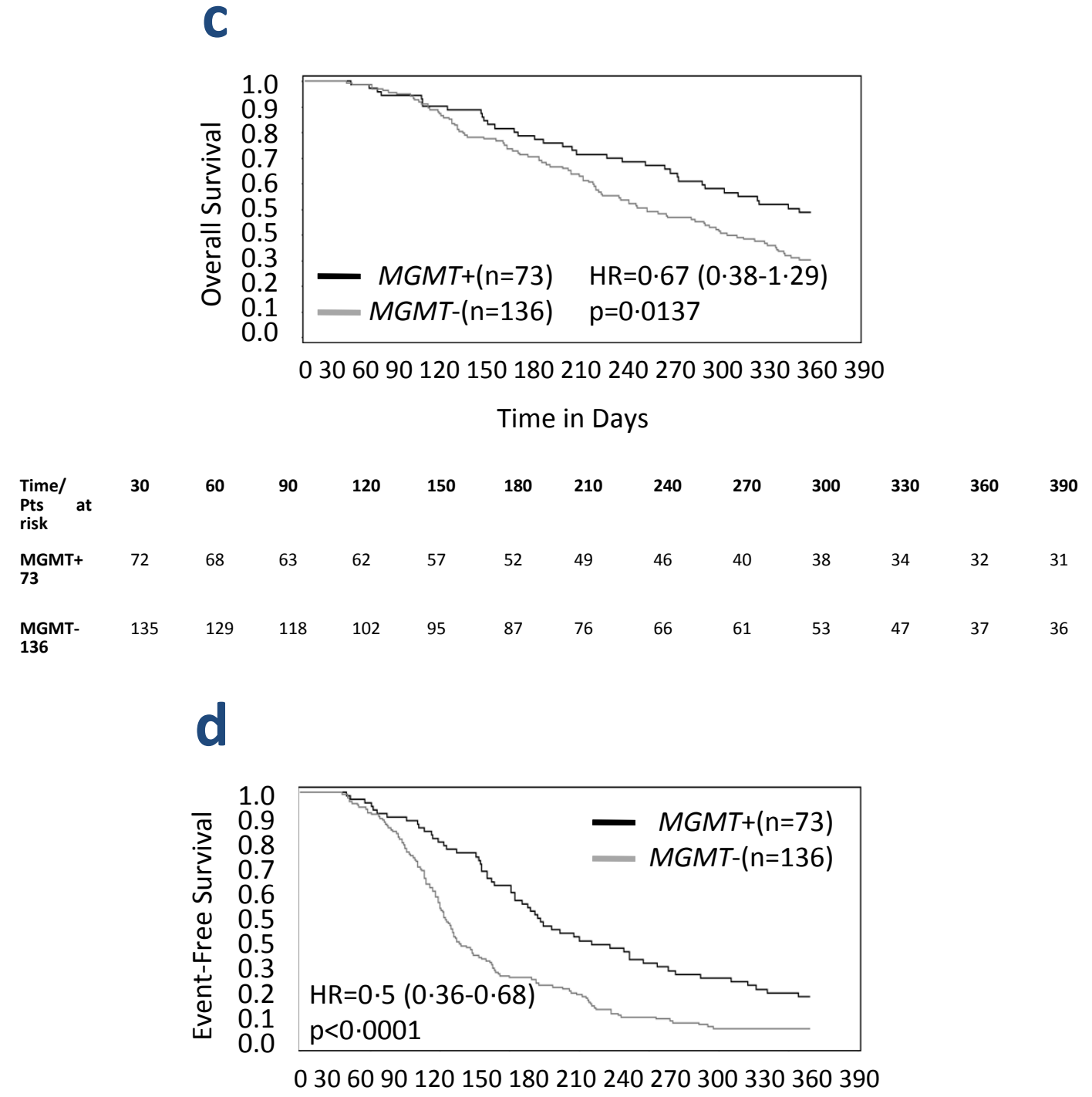

Time in Days

\begin{tabular}{|c|c|c|c|c|c|c|c|c|c|c|c|c|}
\hline $\begin{array}{l}\text { Time/ } \\
\text { Pts } \\
\text { risk }\end{array}$ at & 30 & 60 & 90 & 120 & 150 & 180 & 210 & 240 & 270 & 300 & 330 & 360 \\
\hline $\begin{array}{l}\text { MGMT+ } \\
73\end{array}$ & 72 & 66 & 59 & 53 & 44 & 31 & 27 & 23 & 19 & 18 & 15 & 13 \\
\hline $\begin{array}{l}\text { MGMT- } \\
136\end{array}$ & 135 & 120 & 88 & 52 & 36 & 32 & 21 & 15 & 12 & 9 & 8 & 7 \\
\hline
\end{tabular}


e

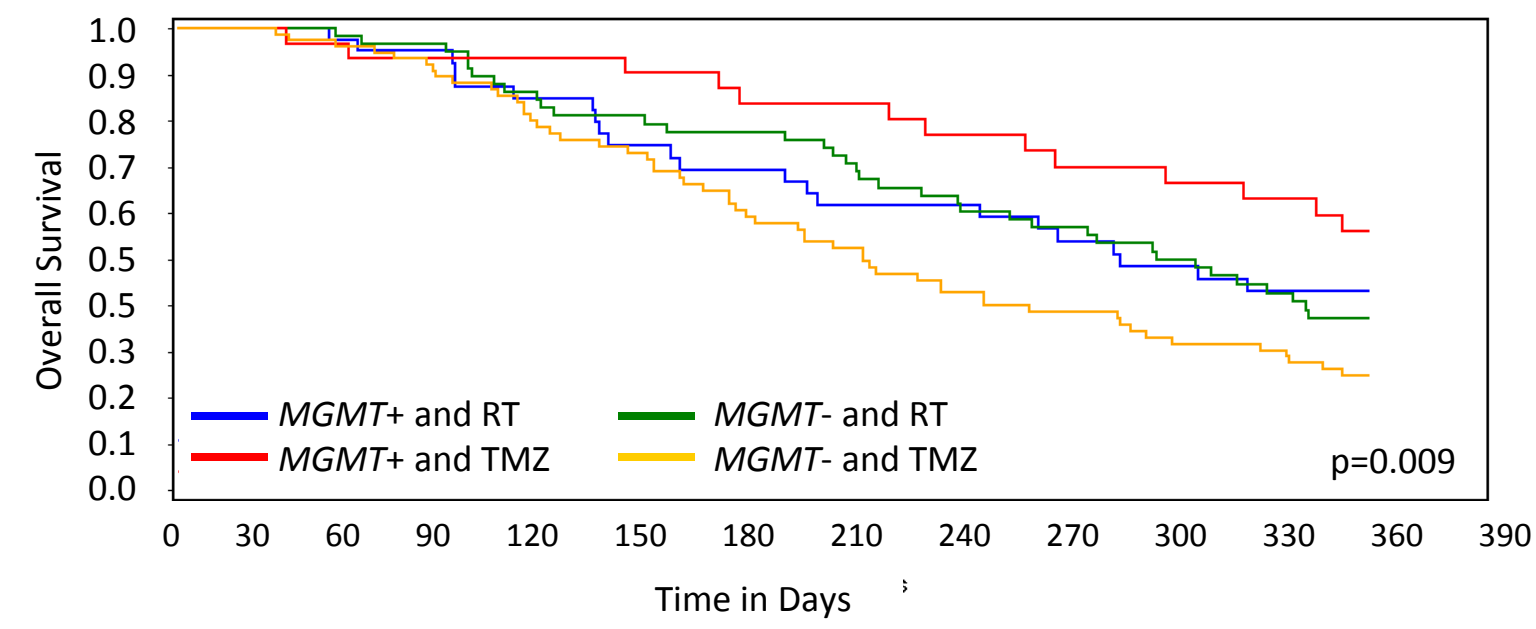

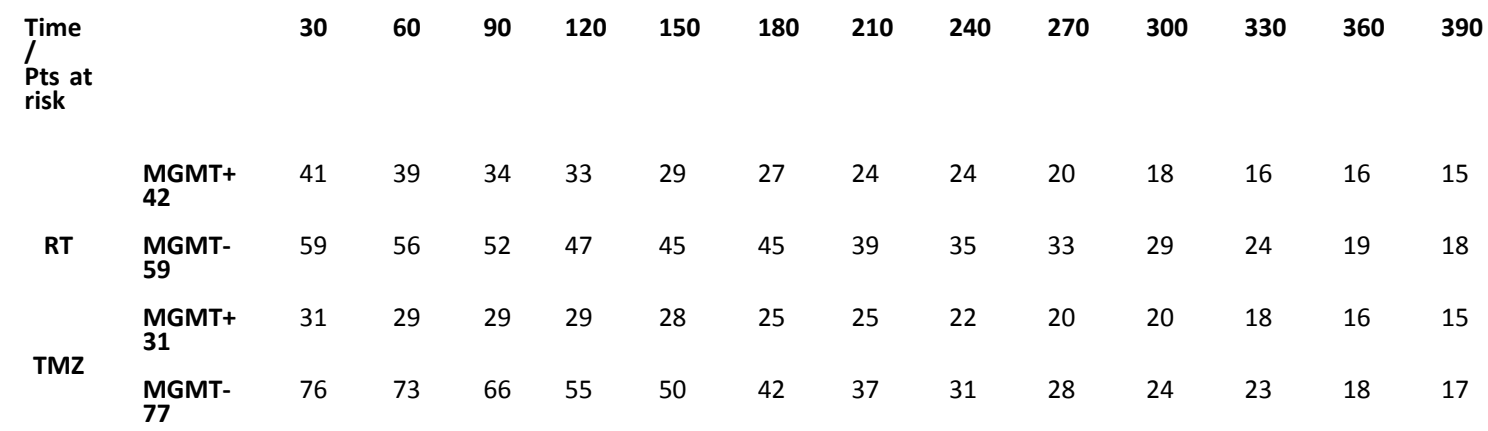

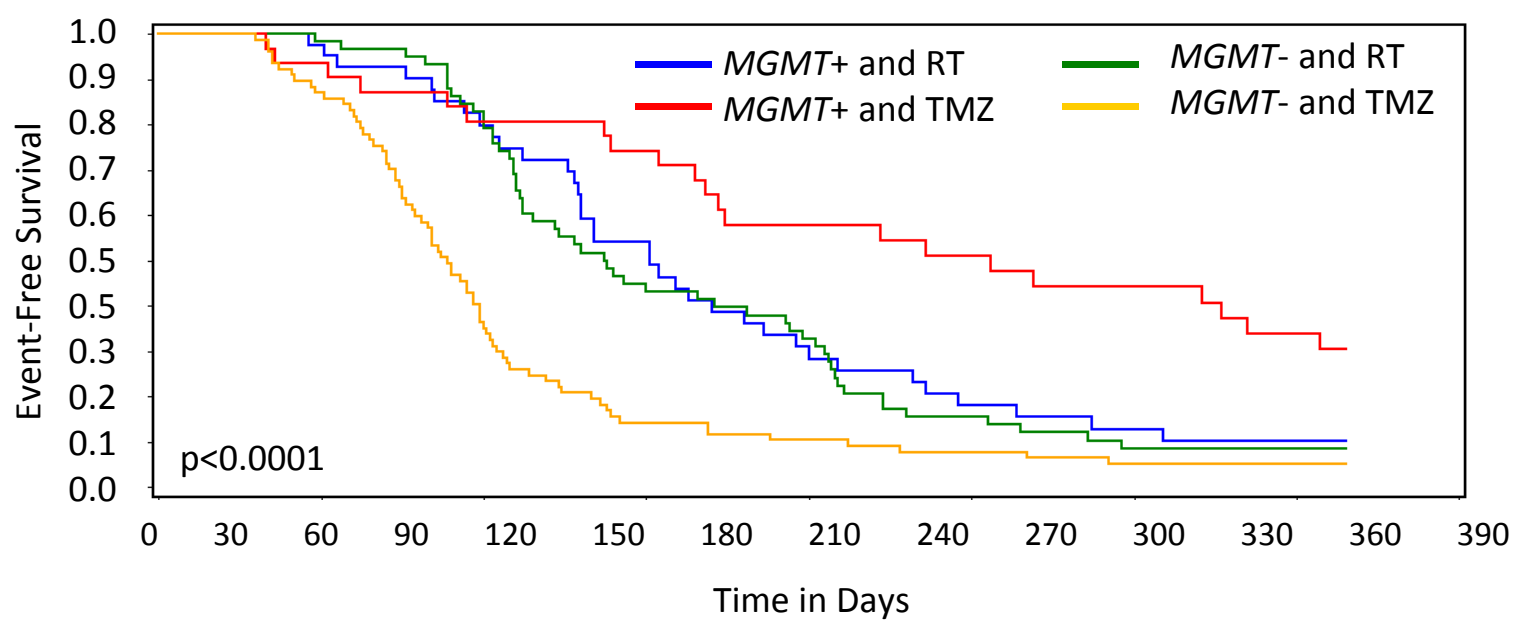

Time
Pts at
risk

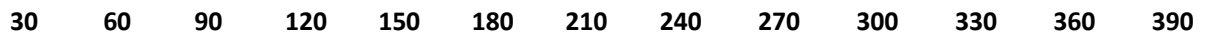

$\begin{array}{lllllllllllllllll} & \begin{array}{l}\text { MGMT+ } \\ \mathbf{4 2}\end{array} & 41 & 38 & 33 & 28 & 21 & 14 & 10 & 8 & 6 & 5 & 4 & 1 & 0 \\ \mathbf{R T} & \begin{array}{l}\text { MGMT- } \\ \mathbf{5 9}\end{array} & 59 & 56 & 50 & 34 & 25 & 23 & 13 & 9 & 7 & 5 & 4 & 1 & 0 \\ & \begin{array}{l}\text { MGMT+ } \\ \mathbf{3 1}\end{array} & 30 & 28 & 26 & 25 & 23 & 17 & 17 & 15 & 13 & 13 & 11 & 9 & 8 \\ \text { TMZ } & \begin{array}{l}\text { MGMT- } \\ \mathbf{7 7}\end{array} & 76 & 63 & 37 & 18 & 11 & 9 & 8 & 6 & 5 & 4 & 4 & 1 & 0\end{array}$


TABLES

Table 1. Baseline Patient Characteristics

\begin{tabular}{|c|c|c|}
\hline & $\begin{array}{c}\text { TMZ } \\
\mathrm{n}=195\end{array}$ & $\begin{array}{c}\mathrm{RT} \\
\mathrm{n}=178\end{array}$ \\
\hline Median age (range), years & $72(66-84)$ & $71(66-82)$ \\
\hline Sex (female/male), $n$ & $107 / 88$ & $90 / 88$ \\
\hline \multicolumn{3}{|l|}{ Central histopathology, n (\%) } \\
\hline AA & $17(9)$ & $23(13)$ \\
\hline GB & $178(91)$ & $153(86)$ \\
\hline Not confirmed & 0 & $2(1)$ \\
\hline Median KPS (range) [\%] & $80(60-100)$ & $80(60-100)$ \\
\hline Prior to treatment & $70(20-100)$ & $80(50-100)$ \\
\hline After primary treatment ${ }^{*}$ & $70(0-100)$ & $70(20-100)$ \\
\hline $\begin{array}{l}\text { Median Mini-Mental State Examination score (out of } 30 \text { ) } \\
\text { (range) }\end{array}$ & $27(9-30)$ & $27(13-30)$ \\
\hline Prior to treatment & $28.5(17-30)$ & $28(12-30)$ \\
\hline After primary treatment ${ }^{*}$ & $28(0-30)$ & $27(11-30)$ \\
\hline \begin{tabular}{|c|} 
Resection, $\mathrm{n}$ \\
Complete \\
Partial \\
Biopsy \\
Missing \\
\end{tabular} & $\begin{array}{c}53 \\
61 \\
80 \\
1 \\
\end{array}$ & $\begin{array}{c}51 \\
62 \\
65 \\
0 \\
\end{array}$ \\
\hline $\begin{array}{l}\text { Steroids, } \mathrm{n} \\
\text { None } \\
\text { At the start of treatment only } \\
\text { At the start and end of treatment } \\
\text { At the end of treatment only } \\
\text { No data }\end{array}$ & $\begin{array}{c}97 \\
8 \\
27 \\
63 \\
0\end{array}$ & $\begin{array}{l}36 \\
26 \\
24 \\
90 \\
2\end{array}$ \\
\hline Duration of treatment (range) [days] & $77(1-1137)$ & $43(1-65)$ \\
\hline $\begin{array}{l}\text { Median time from surgery to start of study treatment (range) } \\
\text { [days] }\end{array}$ & $19(4-47)$ & $30.5(11-76)$ \\
\hline \begin{tabular}{|l|} 
MGMT promoter, $\mathrm{n}$ \\
Methylated \\
Unmethylated \\
Missing/inconclusive \\
\end{tabular} & $\begin{array}{l}31 \\
77 \\
87\end{array}$ & $\begin{array}{l}42 \\
59 \\
77\end{array}$ \\
\hline
\end{tabular}

*Determined at first assessment post RT or the first 3 monthly assessment in the TMZ arm, which are both approximately 3 months after randomization. 
Table 2. Toxicity: Distinct events as recorded in the AE documentation. A new AE in the same category was counted here if the grade of the prior AE of the same kind had returned to CTCAE grade $\leq 1$

\begin{tabular}{|c|c|c|c|c|c|c|}
\hline \multirow[b]{2}{*}{ CTCAE grade } & \multicolumn{3}{|c|}{$\begin{array}{c}\text { TMZ } \\
\mathrm{n}=195\end{array}$} & \multicolumn{3}{|c|}{$\begin{array}{c}\mathrm{RT} \\
\mathrm{n}=178\end{array}$} \\
\hline & 2 & 3 & 4 & 2 & 3 & 4 \\
\hline \multicolumn{7}{|l|}{ Haematological toxicity, $\mathrm{n}$} \\
\hline Neutropenia & 56 & 12 & 4 & 0 & 2 & 0 \\
\hline \multirow{2}{*}{$\begin{array}{l}\text { Lymphocytopenıa } \\
\text { Thrombocytopenia }\end{array}$} & 60 & 44 & 2 & 4 & 1 & 0 \\
\hline & 36 & 12 & 2 & 5 & 4 & 0 \\
\hline Liver enzyme elevation, $\mathrm{n}$ & 42 & 26 & 4 & 13 & 12 & 4 \\
\hline Infection, $\mathrm{n}$ & 54 & 26 & 9 & 40 & 15 & 8 \\
\hline Thrombembolic event, $\mathrm{n}$ & 16 & 18 & 6 & 10 & 4 & 4 \\
\hline Asthenia / Fatigue, $\mathrm{n}$ & 37 & 21 & 3 & 23 & 14 & 6 \\
\hline Nausea / Vomiting, $\mathrm{n}$ & 32 & 6 & 0 & 6 & 1 & 0 \\
\hline Weight loss / Inappetence, n & 8 & 2 & 0 & 2 & 0 & 0 \\
\hline Neurologic symptoms & 73 & 27 & 9 & 31 & 18 & 7 \\
\hline Seizures & 14 & 15 & 2 & 9 & 7 & 6 \\
\hline $\begin{array}{l}\text { Cutaneous } A E \text { (dermatitis, } \\
\text { allergic rash, alopecia), } n\end{array}$ & 15 & 1 & 0 & 18 & 1 & 0 \\
\hline
\end{tabular}




\begin{tabular}{|c|c|c|}
\hline & $\begin{array}{c}\text { TMZ } \\
\mathbf{n}=195 \\
(95 \%-C l)\end{array}$ & $\begin{array}{c}\text { RT } \\
\mathbf{n = 1 7 8} \\
(95 \%-\mathrm{Cl})\end{array}$ \\
\hline Median EFS, HR & \multicolumn{2}{|c|}{$1.15(0.92-1.43)$} \\
\hline Median EFS, months & $3.3(3.2-4 \cdot 1)$ & $4 \cdot 7(4 \cdot 2-5 \cdot 2)$ \\
\hline EFS rate at 6 months, $\%$ & $30 \cdot 1(23 \cdot 6-36 \cdot 6)$ & $35.1(28.0-42 \cdot 3)$ \\
\hline EFS rate at 12 months, $\%$ & $12 \cdot 0(7 \cdot 9-17 \cdot 1)$ & $9.3(5 \cdot 5-14 \cdot 2)$ \\
\hline Median OS, HR & \multicolumn{2}{|c|}{$1.09(0.84-1.42)$} \\
\hline Median OS, months & $8 \cdot 6(7 \cdot 3-10 \cdot 2)$ & $9.6(8 \cdot 2-10 \cdot 8)$ \\
\hline OS at 6 months, $\%$ & $66 \cdot 7(60 \cdot 0-73 \cdot 4)$ & $71.7(65.0-78.4)$ \\
\hline OS at 12 months, $\%$ & $34.4(27 \cdot 6-41 \cdot 4)$ & $37 \cdot 4(30 \cdot 1-44 \cdot 7)$ \\
\hline
\end{tabular}


Table 4a. Prognostic and predictive factors as determined in a multivariate Coxregression analysis for the primary endpoint OS ( $n=208$ from 373$)^{*}$.

\begin{tabular}{|l|c|c|}
\hline & Hazard ratio (95\% CI) & P value \\
\hline Age (years) & $1.02(0.98-1.06)$ & 0.285 \\
\hline $\begin{array}{l}\text { Resection } \\
\text { Complete versus incomplete versus biopsy }\end{array}$ & $1.84(1.44-2.35)$ & $<0.0001$ \\
\hline AA versus GB & $0.69(0.38-1.22)$ & 0.201 \\
\hline TMZ, MGMT methylated & $0.69(0.35-1.16)$ & 0.139 \\
RT, MGMT methylated and unmethylated & $1.0($ Reference) & \\
TMZ, MGMT unmethylated (95\%-CI) & $1.34(0.92-1.95)$ & 0.129 \\
\hline
\end{tabular}

Table 4b. Prognostic and predictive factors as determined in a multivariate Coxregression analysis for EFS ( $n=208$ from 373$)^{*}$.

\begin{tabular}{|l|c|c|}
\hline & Hazard ratio (95\% CI) & P value \\
\hline Age (years) & $1.01(0.98-1.04)$ & 0.674 \\
\hline $\begin{array}{l}\text { Resection } \\
\text { Complete versus incomplete versus biopsy }\end{array}$ & $1.29(1.07-1.56)$ & 0.008 \\
\hline AA versus GB & $0.75(0.45-1.24)$ & 0.255 \\
\hline TMZ, MGMT methylated & $0.53(0.33-0.86)$ & 0.01 \\
RT, methylated and unmethylated & $1.0($ Reference) & \\
TMZ, MGMT unmethylated & $1.95(1.41-2.69)$ & 0.01 \\
\hline
\end{tabular}

* 164 without methylation status, 1 without resection status 